\title{
Treating depression in the Caregivers of Cancer Patients
}

\begin{abstract}
Partners often assume the role of caregiver when cancer is brought into the home. The support caregivers provide to cancer patients has immeasurable value. However, care giving can take a significant toll on loved ones from both a psychological and physiological perspective; sometimes to the point of producing caregiver burden. One of the diseases most prevalent in caregivers is depression. Signs and symptoms of depression amongst caregivers vary from mild to severe and people respond quite differently to the life stressors they face. While, the exact cause of depression is not known, proven research correlates it with an internal chemical and/or neurotransmitter imbalance that can be triggered by stress, chronic stress in particular. As a mood disorder, depression affects how you feel, think, and function in the performance of your daily activities. It is critically important to identify caregivers exhibiting depressive symptoms and first, to provide them with both emotional and psychological support, and second, so as to ensure the individual battling cancer receives optimal support from their loved ones. This review article serves to promote health awareness and the unmet needs of cancer patient caregivers, the presence of caregiver burden, and the impact that stress can have on one's neurophysiological processes to precipitate the development of mental illness such as depression.
\end{abstract}

Keywords: cancer, caregivers, psychological adjustment, depression, partner, informal caregiver, unmet needs
Volume 7 Issue 5 - 2017

\author{
Joyce M Louis,' Lisa Adams,' Tony L Brown² \\ 'USAT College of Medicine, USA \\ ${ }^{2}$ Harvard University, USA
}

Correspondence: Joyce M. Louis, Major General Hugh G.

Robinson Center for Neuropsychiatric Studies, USA,

Email author@robinsoncns.info

Received: April 0I, 2017 | Published: April 25, 2017
Abbreviations: HPA, hypothalamic-pituitary-adrenal; SNS, sympathetic nervous system; CRH, corticotropin-releasing hormone; $\mathrm{ACTH}$, adrenocorticotropic hormone

\section{Introduction}

According to the National Cancer Institute, ${ }^{1}$ it was estimated that $1,685,210$ new cases of cancer would be diagnosed in the United States in 2016 and about 595,690 people will have died from the disease. The incidence of new cases of cancer is reportedly 454.8 per 100,000 men and women per year (based on 2008-2012 cases). Further, the number of people living beyond a cancer diagnosis reached nearly 14.5 million in 2014 and is expected to reach 19 million by 2024 . According to the 2016 Cancer Treatment and Survivorship Statistics report, one-third of cancer survivors in the United States today were diagnosed less than five years, ${ }^{2}$ with 14.5 million cancer survivors now living in the U.S. ${ }^{2}$ Receiving a diagnosis of cancer is life-changing and traumatic for all involved. As the prevalence of cancer continues to rise and cause considerable burden worldwide, such as mortality, years of life lost, and years lived with disability, it comes as no surprise to say cancer is the second leading cause of death in the United States.

Cancer not only takes a toll on the quality of the lives and functioning of individuals who are diagnosed with and dealing with cancer, but also on the care givers of these individuals. Caregivers, also referred as informal caregivers, are people (most commonly partners or spouses) who provide essential support to cancer patients throughout the trajectory of the illness. These individuals offer practical help, psychological support, emotional help, and sometimes assistance with everyday life activities such as bathing, dressing and feeding. Although caregivers complain little, they often experience a great deal of uncertainty, concern and lack of hope. As found by Bevans \& Sternberg, ${ }^{3}$ caregivers often report severe emotional distress, significant fatigue, sleep impairment, and difficulty maintaining their focus and energy throughout the cancer treatment process of their loved ones; many symptoms of which characterize depression. Sternberg et al., ${ }^{4}$ add that worry, anxiety and loneliness also occur. However, when the health of the caregiver suffers, the impact becomes manifested in the person for which the care is provided. It is the purpose of this paper to explore the impact of stress on the health of informal cancer caregivers who support and attend to the needs of those suffering from cancer; paying particular attention to the presence of depression.

\section{Discussion}

Cancer not only affects those in receipt of the diagnosis, but also their caregivers. Most cancer treatment takes place in outpatient settings, ${ }^{5}$ giving partners more responsibilities; particularly providing around the clock constant care. Many studies are now beginning to focus on a family perspective to cancer, which raises the growing interest of unmet needs of caregivers. It appears that cancer caregivers can have considerably more unmet needs than the patients they care for. ${ }^{6}$ Partners of cancer patients are at increased risk of depression and other related factors.

Depression in caregivers is a specific emotional reaction to the stress of caregiving. Studies have found that $32 \%-50 \%$ of caregivers had depressive symptoms at a level suggesting clinical depression. ${ }^{7,8}$ One study by Sklenarova et al., ${ }^{6}$ revealed that caregivers exhibited higher anxiety and distress compared with actual cancer patients and $43.6 \%$ had needs that were unmet. ${ }^{6}$ High levels of stress have been associated with psychological and physiological disturbances in the partners of patients with cancer. ${ }^{9}$

Assessing individuals with depressive symptoms may help to identify individuals who are experiencing a dysfunctional grief pattern that may lead to higher levels of depression..$^{10}$ The identification of depression therefore is important to relieve caregiver suffering during and after the caregiving experience. ${ }^{11}$ 
The experience of caring for an individual who is suffering with cancer and undergoing cancer treatments is inherently stressful and one's perception of the event plays a significant role in the body's response. Stress exerts many effects on various organs of the body, particularly, the brain's neurological and mental aspects, but also clinically, survivorship of loved ones is also gaining ground.

\section{Redefining survivorship}

As identified by the Center of Disease Control (2004), survivorship now encompasses, not just the survivor of the cancer, but the family and loved ones as well. They state "for many years, the focus of cancer diagnosis and treatment was on the person diagnosed with the disease. However, recent advances in our understanding of survivorship have led to the expanded definition of "survivor" to include others touched by this disease, such as families, friends, and caregivers (p.7)."

The burden of care giving is phenomenal, particularly when that care is informally provided. A loved one or secondary survivor, as sometimes referred to, has an incredibly difficult task in providing support to loved ones during and in the aftermath of cancer treatment and recovery. A secondary survivor did not physically experience the battle with cancer but were, nonetheless, impacted by the traumatic experiences their partner had to endure during treatment. For some couples, challenging cancer together can potentially strengthen their relationship and commitment, but for others, cancer may create new problems. Long-term partnerships can become affected after the diagnosis of cancer as both individuals may experience depression.

In all aspects of health, whether it relates to one's mental, physical, social or financial well-being, caregiver burden is no stranger. This experience is commonly perceived as a chronic stressor, and caregivers often experience negative psychological, behavioral, and physiological effects on their daily lives and health. ${ }^{3}$ Often the partner is too concerned and preoccupied with providing adequate care and support to an ill partner to perceive his/her own health or lack thereof and seek help. Too frequently we overlook the spouse's vital role in staying alongside a cancer patient. Awareness of caregiver depression is important because of its impact on health deterioration and early death in caregivers. Studies have suggested that caregiver depression may result from anticipatory grieving of losses resulting from the partner's illness as well as the eventual death of the partner. ${ }^{12}$

The mental impact a caregiver endures is individualized, however, the threat remains that it can potentially precipitate the development of various psychiatric disorders such as depression, anxiety and addictions. ${ }^{8,13}$ Hence, informal caregivers are increasingly susceptible to mental and emotional challenges. ${ }^{14-16}$ Long-term sequelae of depression embody accumulated levels of coronary heart disease and metabolic syndrome when compared to non-caregivers. ${ }^{17}$ First, you bear witness to your loved one fighting the biggest battle of their life and deteriorating right in front of your eyes. The person you once knew as a husband or wife for example, is changing, literally, in every sense of the word inclusive of their energy, spirit, overall health and self-care abilities. As the person you once knew changes with declining health, you literally experience the stages of grieving for the individual even though they are physically alive. Hence, working one's way through Kubler-Ross' stages of grieving, inclusive of denial, anger, bargaining, depression and acceptance, is not uncommon. ${ }^{13}$

A further paradoxical twist also occurs for the individual suffering with the cancer. The mental health of cancer patients, can reciprocally, be affected by the emotional health of their caregivers. Caregiver depression, for example, may reveal patient depression, ${ }^{12}$ therefore only adding to the stressors already experienced in one's battle with cancer.

\section{The neurophysiological effects of stress on caregivers}

Caregiver health is critical, particularly when one is performing the role of primary caregiver. When caregiver health is jeopardized, one is of little help to themselves much less taking the lives of others into their hands. As tough economic times and fiscal restraints require two household income earners to survive, ${ }^{18}$ the adopted role of one earner as a primary caregiver exerts even more family and/or household pressure. In addition to financial hardship, informal care giving by family members drains them of their mental, emotional, physical, and social supports as well. ${ }^{19}$

The impact of stress on the brain significantly contributes to the development of mental illnesses in general or at least to enrich the foundation on which they precipitate. With the brain as a starting point, very soon the effect of long term stress begins to permeate systemically into other body organs as well. Through the sympathetic nervous system pathway, every organ in the body is affected by yet another hormone, adrenalin that gets released once stress is experienced. ${ }^{20}$ The death and dying of neurons is how stress often impacts the brain.

All stressors activate the hypothalamic-pituitary-adrenal (HPA) axis and the adrenergic sympathetic nervous system (SNS). Following exposure to stressful stimuli, the hypothalamus releases corticotropinreleasing hormone $(\mathrm{CRH})$, which then stimulates the pituitary gland to release adrenocorticotropic hormone $(\mathrm{ACTH})$. ACTH in turn triggers the release of glucocorticoids (cortisol) from the adrenal cortex. At the same time the sympathetic nervous system is activated with release of epinephrine (adrenalin) from the adrenal medulla and norepinephrine from sympathetic nerves. In combination, the molecules released during the stress response have profound effects on the function of most cells and organs throughout the body, including the brain, heart, respiratory system, muscles, skin, liver, gastrointestinal tract and, importantly, the immune system. The death of neurons and neuronal atrophy that occurs in the limbic, hypothalamic, and even the whole prefrontal cortex can shrink significantly in size, infringing on one's memory and learning process ability. Its neuronal synapses and synaptic activity are all compromised potentially causing increased aging of the brain. In addition, with glucocorticoid hormones as one of the key escalating mediators in this process can actually cause the death of neurons. ${ }^{21}$ This steady release of Cortisol precipitated by stress, for example, causes the brain to become battered; ${ }^{22}$ leading not only to a great deal of wear and tear on the brain, ${ }^{23}$ but literally excites the neurons to death; structurally altering the brain.

Ongoing stress, particularly when it becomes chronic, creates a metamorphosis of the brain where remodelling and/or modifications of the brain. ${ }^{23}$ These modifications create smaller brain structures, in particular, a smaller hippocampus results in cognitive impairments, prolonged depression and decreased self-esteem. ${ }^{23}$ Further, psychological stress, specifically, modulates cytokines production. While cytokines are responsible for regulatory central nervous system functions and hence resulting behaviors, the potential relevance of cytokines to one's mental health is strongly correlated..$^{24}$ Over time stress causes disruptions to neural circuitry, neurotransmitter metabolism, neuro-endocrine function and synaptic plasticity. ${ }^{24}$ What results is dysfunctional cytokine signaling, neuro-inflammation and aberrant behaviors affiliated with potential outcomes of depression, anxiety and cognitive dysfunction. 
Caregiver burden is closely linked to ongoing chronic stress and its underlying physiology. In "chronic stress the healthy 'diurnal rhythm' of the HPA axis, with a late evening nadir and a morning peak, is flattened showing instead low morning and elevated afternoon/evening cortisol levels". ${ }^{25}$ This is what best represents a state of physiological "burn-out" that often accompanies psychological burnout comparable to that of caregiver burden. Although HPA axis activity is increased initially, it decreases over time. At this point, informal caregivers of cancer patients show an autonomic imbalance with a shift towards the sympathetic and away from the parasympathetic tone. ${ }^{26,27}$ This blunted HPA axis response and functional glucocorticoid resistance is what is associated with pro-inflammatory states that predisposes a caregiver to autoimmune/inflammatory diseases, ${ }^{5,28}$ cardiovascular disease, ${ }^{29}$ and yes, depression. ${ }^{22}$

\section{Clinical manifestations of depression in caregivers}

Depression is related to an impaired quality of life and ability to engage in social interactions, interpersonal relationships, and activities of independent living. ${ }^{30}$ It is believed that depression is often caused by the influence of more than a single factor. ${ }^{31}$ Depression can be triggered by genetic influence, psychological influence, biological influence, medical illness, environmental influence, or other causes. ${ }^{31}$ Although well researched and prevalent, most individuals with depression do not seek care and those who seek care often feel as though they have not fully recovered.

Being despondent is not a sign of depression. Mood can vary with the broad range of normal emotions that people experience. When your mood becomes patent for an extended period of time, depression can correctly be diagnosed. ${ }^{32}$ Some people may experience every symptom, many, or few of the common symptoms of depression. Symptoms and how long they last depend on the individual and their particular illness or influence.

Suffering from depression is identifying with several of the following signs and symptoms. Although many of these symptoms listed can be a part of everyday life, the stronger they become and the longer they've lasted, the more probable it is that you're dealing with depression..$^{33}$ Some of these symptoms are allows for depression to be accepted as the conventional diagnosis: persistent feelings of helplessness or pessimism, loss of interest or pleasure in hobbies, irritability, appetite and sleep changes, loss of energy, difficulty concentrating, self-loathing, idiopathic aches or pains, or suicidal thoughts or attempts. ${ }^{33}$

\section{Services for partners}

To be a good caregiver, you must be good to yourself. The most general feelings of stress, anxiety, and depression felt by caregivers are the feeling of being overwhelmed or burned out. Learning the signs of stress and seeking help from others is the best way to cope with depression. Further, finding effective coping strategies such as exercise, is yet a critical factor in allaying some of the stress encountered. Finding a support group, asking questions to an oncology social worker or psychologist, or hiring people to assist with caring for the person with cancer are a few suggestions.

A few other ways to help manage depression include: avoiding excessive alcohol, maintaining supportive relationships by making time for yourself and other relationships, peer cancer support groups, doing activities that bring you happiness and comfort, and practicing relaxation techniques. Psychotherapy such as cognitive-behavior therapy also significantly treats depression..$^{32}$ Restoring balance through eating mood-boosting diets or cultivating self-love and acceptance is also helpful..$^{32}$ As no two people are alike, some trial and error may be anticipated to find the best suitable treatment.

Pharmacological interventions are also advisable for individuals experiencing significant changes of life and/or events. Depressive symptoms can be moderated with antidepressant, which enhances monoamine neurotransmission ${ }^{34}$ and serotonin levels. ${ }^{35}$ Recent studies suggest that depression starts with the formation and function of neurons, higher up than simple chemicals. In other words, monoamines are most likely not the only factor diving the clinical presentation of depression. ${ }^{34}$ While some reviews suggest that antidepressants are only marginally more effective than placebos in terms of improvements in mood, ${ }^{36}$ more recently, the advent of selective serotonin-reuptake inhibitors (SSRIs), has been met with tremendous success in enabling an individual to live and deal with their depression. ${ }^{35-38}$

\section{Conclusion}

Being a caregiver to a loved one who is battling cancer is a difficult, onerous, and potentially, life changing event. Caregiver burden manifests itself in many ways, impacting one's physical, emotional, mental and physiological health. The mental health of a caregiver is particularly concerning as multiple stressors often occur. Depression, for example, is often directly related to a significant event such as battling an illness or losing a loved one. An important task that caregivers often neglect is caring for themselves. A caregiver's mental, physical and emotional health is not only integral to themselves as functioning and healthy support persons, but also for the well-being of the person who is receiving treatment for cancer. It is important that medical professionals involved in the care of cancer patients be aware of the demands made on caregivers. Caregiver burden is a prime predictor of depression among cancer patient caregivers, hence interventions aimed at reducing the psychiatric effects of cancer ought to focus on the patient as well as the caregiver, where a holistic family care approach ensues.

\section{Acknowledgments}

None.

\section{Conflicts of interest}

Author declares there are no conflicts of interest.

\section{Funding}

None.

\section{References}

1. American Cancer Society. Cancer: About us, who we are and our history. 2015.

2. American Cancer Society. Cancer survivors: A growing population: New report projects 20 million cancer survivors in the US by 2026 Science Daily. 2016

3. Bevans MF, Sternberg EM. Caregiving Burden, Stress, and Health Effects among Family Caregivers of Adult Cancer Patients. JAMA. 2012;307(4):398-403.

4. Stenberg U, Ruland CM, Miaskowski C. Review of the literature on the effects of caring for a patient with cancer. Psychooncology. 2010;19(10):1013-1025. 
5. Vallerand AH, Collins-Bohler D, Templin T, et al. Knowledge of and barriers to pain management in caregivers of cancer patients receiving homecare. Cancer Nurs. 2007;30(1):31-37.

6. Sklenarova H, Krümpelmann A, Haun MW, et al. When do we need to care about the caregiver? Supportive care needs, anxiety, and depression among informal caregivers of patients with cancer and cancer survivors. Cancer. 2015;121(9):1513-1519.

7. Butler SS, Turner W, Kaye LW, et al. Depression and caregiver burden among rural elder caregivers. J Gerontol Soc Work. 2005;46(1):56.

8. Covinsky KE, Newcomer R, Fox P, et al. Patient and caregiver characteristics associated with depression in caregivers of patients with dementia. J Gen Intern Med. 2003;18(12):1006-1014.

9. Gregorio SW, Carpenter KM, Dorfman CS, et al. Impact of breast cancer recurrence and cancer-specific stress on spouse health and immune function. Brain Behav Immun. 2012;26(2):228-233.

10. Grassi L. Bereavement in families with relatives dying of cancer. Curr Opin Support Palliat Care. 2007;1(1):43-49.

11. Rivera, H.R. Depression symptoms in cancer caregivers. Clinical Journal of Oncology Nursing. 2009;13(2):195-202.

12. Lindemann E. Symptomatology and management of acute grief. American Journal of Psychiatry. 1994;151(Suppl 6):155-160.

13. Warchol-Biedermann K, Mojs E, Gregersen R, et al. What causes grief in dementia caregivers. Arch Gerontol Geriatr. 2014;59(2):462-467.

14. Clay OJ, Roth DL, Wadley VG, et al. Changes in social support and their impact on psychosocial outcome over a 5-year period for African American and White dementia caregivers. Int $J$ Geriatr Psychiatry. 2008;23(8):857-862.

15. Fisher GG, Franks MM, Plassman BL, et al. Caring for individuals with dementia and cognitive impairment, not dementia: findings from the Aging, Demographics, and Memory Study. J Am Geriatr Soc . 2011;59(3):488-494.

16. Sörensen S, Conwell Y. Issues in dementia caregiving: effects on mental and physical health, intervention strategies, and research needs. Am J Geriatr Psychiatry. 2011;19(6):491-496.

17. Vitaliano PP, Scanlan JM, Zhang J, et al. A path model of chronic stress, the metabolic syndrome, and coronary heart disease. Psychosom Med. 2002;64(3):418-435.

18. Duxbury L, Higgins C, Schroeder B. Balancing Paid Work and Caregiving Responsibilities: A Closer Look at Family Caregivers in Canada. Human Resources and Skills Development Canada. 2009.

19. McCann TV, Bamberg J, McCann F. Family carers' experience of caring for an older parent with severe and persistent mental illness. Int J Ment Health Nurs. 2015;24(3):203-212.

20. Fuchs E, Flügge G. Chronic social stress: Effects on limbic brain structures. Physiol Behav. 2003;79(3):417-27.
21. Fuchs E, Flügge G. Stress, glucocorticoids and structural plasticity of the hippocampus. Neurosci Biobehav Rev. 1998;23(2):295-300.

22. Pace TWW, Hu F, Miller AH. Cytokine-effects on glucocorticoid receptor function: Relevance to glucocorticoid resistance and the pathophysiology and treatment of major depression. Brain Behav Immun. 2007;21(1):9-19.

23. MacEwan BS. Physiology and neurobiology of stress and adaptation: Central role of the brain. Physiol Rev. 2007;87(3):873-904.

24. Salim S, Chugh G, Asghar M. Inflammation in anxiety. Adv Protein Chem Struct Biol. 2012;88:1-25.

25. Heim C, Ehlert U, Hellhammer DH. The potential role of hypocortisolism in the pathophysiology of stress-related bodily disorders. Psychoneuroendocrinology. 2000;25(1):1-35.

26. Lucini D, Cannone V, Malacarne M, et al. Evidence of autonomic dysregulation in otherwise healthy cancer caregivers: a possible link with health hazard. Eur J Cancer. 2008;44(16):2437-2443.

27. Rohleder N, Marin TJ, Ma R, et al. Biologic Cost of Caring for a Cancer Patient: Dysregulation of Pro- and Anti-Inflammatory Signaling Pathways. J Clin Oncol. 2008;27(18):2909-2915.

28. Sternberg EM. Neural regulation of innate immunity: a coordinated nonspecific host response to pathogens. Nat Rev Immunol. 2006;6(4):318-328.

29. Nijm J, Jonassonm L. Inflammation and cortisol response in coronary artery disease. Annals of Medicine. 2009;41(3):224-233.

30. Sobocki P, Ekman M, Agren H, et al. Health-related quality of life measured with EQ-5D in patients treated for depression in primary care. Value Health. 2007;10(2):153-160.

31. Tully EC, Iacono WG, Mc Gue M. Changes in Genetic and Environmental Influences on the Development of Nicotine Dependence and Major Depressive Disorder from Middle Adolescence to Early Adulthood. Development and Psychopathology. 2010;22(4):831-848.

32. Chopra D. Healing from Depression. 2015.

33. National Institute of Health. Depression. Bethesda, MD: U.S. Government Printing Office. 2015.

34. Delgado PL. How Antidepressants Help Depression: Mechanisms of Action and Clinical Response. J Clin Psychiatry. 2004;65(Suppl 4):25-30.

35. Mayo Clinic. Selective serotonin reuptake inhibitors. 2017.

36. Moncrieff J, Wessely S, Hardy R. Active placebos versus antidepressants for depression. Cochrane Database Syst Rev. 2004;1:CD003012.

37. Good fellow LM. Effects of therapeutic back massage on psychophysiologic variables and immune function in spouses of patients with cancer. Nurs Res. 2003;52(5):318-328.

38. National Cancer Institute. About cancer: statistics. 2016. 